Dokuz Eylül Üniversitesi-Mühendislik Fakültesi

Fen ve Mühendislik Dergisi

Cilt 19, Sayı 57, Eylül 2017
Dokuz Eylul University-Faculty of Engineering Journal of Science and Engineering Volume 19, Issue 57, September 2017

DOI: $10.21205 /$ deufmd.2017195764

\title{
Spacelike Regle Yüzeylerin Frenet Çatıları ve Frenet İnvaryantları
}

\author{
Mehmet ÖNDER $^{* 1}$, Hasan Hüseyin UĞURLU ${ }^{2}$ \\ ${ }^{1}$ Bağımsız Araştırmacı, Delibekirli Mahallesi, Tepe Sokak, No: 63, 31440, Kırıkhan, \\ Hatay \\ ${ }^{2}$ Gazi Üniversitesi, Gazi Eğitim Fakültesi, Orta Öğretim Fen ve Matematik Alanları \\ Eğitimi Bölümü, 06500, Ankara
}

(Alınış / Received: 06.04.2016, Kabul / Accepted: 28.06.2017, Online Yayınlanma / Published Online: 20.09.2017)

\begin{abstract}
Anahtar Kelimeler Minkowski 3uzayı, Spacelike regle yüzey, Frenet çatısı

Özet: Bu çalışmada spacelike regle yüzeyler için Chasles teoremi sunulmuş ve bir spacelike regle yüzey ile bu yüzeyin yönlü konisinin Frenet çatıları ve invaryantları verilmiștir. Bir spacelike regle yüzey ile bu yüzeyin yönlü konisinin aynı Frenet çatısına sahip olduğu gösterilmiştir.
\end{abstract}

\section{Frenet Frames and Frenet Invariants of Spacelike Ruled Surfaces}

\begin{tabular}{ll}
\hline Keywords & Abstract: In this study, we introduce the Chasles theorem for \\
Minkowski 3- & spacelike ruled surfaces and give the Frenet frames and invariants \\
space, & of a spacelike ruled surface and of its directing cone. We show that \\
Spacelike ruled & a spacelike ruled surface and its directing cone have the same \\
surface, & Frenet frame.
\end{tabular}

*Corresponding author: mehmetonder197999@gmail.com

\section{Introduction}

A ruled surface is a special surface generated by moving a straight line continuously in the space. Since the ruled surfaces have important positions and applications in study of design problems in spatial mechanisms and physics, these surfaces are one of the most important topics of surface theory. Because of this position of the ruled surfaces, many geometers have studied on them in the Euclidean space and they have investigated many properties of the ruled surfaces $[6,7,12,14]$. Furthermore, the differential geometry of the ruled surfaces in Minkowski space has been studied by several authors [2$4,8,9,11,15]$.
In the Euclidean 3-space $E^{3}$, the Frenet frames and formulas of a ruled surface have been introduced by Karger and Novak [7]. Frenet frames and Frenet invariants of a ruled surface have large applications in mechanics and kinematics. For instance, the kinematic differential geometry of a rigid body is based on the frames and invariants of ruled surfaces. Instantaneous properties of a point trajectory and of a line trajectory in spatial kinematics have been studied by Wang, Liu and Xiao. They have also obtained the Euler-Savary analogue equations of a point trajectory and of a line trajectory $[17,18]$. Furthermore, they have given the distributions of characteristic lines in the moving body in spatial motion [19]. 
Moreover, Önder and Uğurlu have introduced the Frenet frames, Frenet invariants and the instantaneous rotation vectors of timelike ruled surfaces in the Minkowski 3-space [11].

In this study, we give the Frenet frame, invariants and instantaneous rotation vector of a spacelike ruled surface in the Minkowski 3-space $I R_{1}^{3}$. We hope that, similar to the Euclidean spatial kinematics, this study lets new studies in Lorentzian spatial kinematics like instantaneous properties of a point trajectory and of a line trajectory in Lorentzian spatial kinematics.

\section{Preliminaries}

The Minkowski 3-space $I R_{1}^{3}$ is the real vector space $I R^{3}$ provided with standard flat metric given by

$$
\langle,\rangle=-d x_{1}^{2}+d x_{2}^{2}+d x_{3}^{2}
$$

where $\left(x_{1}, x_{2}, x_{3}\right)$ is a standard rectangular coordinate system of $I R_{1}^{3}$. An arbitrary vector $\vec{v}=\left(v_{1}, v_{2}, v_{3}\right)$ in $I R_{1}^{3}$ can have one of three Lorentzian causal characters; it can be spacelike if $\langle\vec{v}, \vec{v}\rangle>0 \quad$ or $\quad \vec{v}=0$, timelike if $\langle\vec{v}, \vec{v}\rangle<0$ and null(lightlike) if $\langle\vec{v}, \vec{v}\rangle=0$ and $\vec{v} \neq 0$. Similarly, the Lorentzian casual character of a curve

$$
\vec{\alpha}=\vec{\alpha}(s): I \subset I R \rightarrow I R_{1}^{3}
$$

is determined by the character of the velocity vector $\vec{\alpha}^{\prime}(s)$ [10]. For a vector $\vec{v} \in I R_{1}^{3}$, the norm function is defined by

$$
\|\vec{v}\|=\sqrt{|\langle\vec{v}, \vec{v}\rangle|} .
$$

For any vectors $\vec{x}=\left(x_{1}, x_{2}, x_{3}\right)$ and $\vec{y}=\left(y_{1}, y_{2}, y_{3}\right)$ in $I R_{1}^{3}$, the cross product of $\vec{x}$ and $\vec{y}$ is given by

$$
\begin{gathered}
\vec{x} \times \vec{y}=\left(x_{2} y_{3}-x_{3} y_{2}, x_{1} y_{3}-x_{3} y_{1},\right. \\
\left.x_{2} y_{1}-x_{1} y_{2}\right)
\end{gathered}
$$

The Lorentzian sphere and hyperbolic sphere of radius $r$ and center origin in $I R_{1}^{3}$ are

$S_{1}^{2}=\left\{\vec{x}=\left(x_{1}, x_{2}, x_{3}\right) \in I R_{1}^{3}:\langle\vec{x}, \vec{x}\rangle=r^{2}\right\}$, and

$H_{0}^{2}=\left\{\vec{x}=\left(x_{1}, x_{2}, x_{3}\right) \in I R_{1}^{3}:\langle\vec{x}, \vec{x}\rangle=-r^{2}\right\}$, respectively [16].

Definition 2.1. ([13]) i) Hyperbolic angle: Let $\vec{x}$ and $\vec{y}$ be timelike vectors in $I R_{1}^{3}$. Then there is a unique real number $\theta$ such that $<\vec{x}, \vec{y}>=-\|\vec{x}\|\|\vec{y}\| \cosh \theta . \quad$ This number is called the hyperbolic angle between the vectors $\vec{x}$ and $\vec{y}$.

ii) Central angle: Let $\vec{x}$ and $\vec{y}$ be spacelike vectors in $I R_{1}^{3}$ that span a timelike vector subspace. Then there is a unique real number $\theta$ such that $|<\vec{x}, \vec{y}\rangle \mid=\|\vec{x}\|\|\vec{y}\| \cosh \theta$. This number is called the central angle between the vectors $\vec{x}$ and $\vec{y}$.

iii) Spacelike angle: Let $\vec{x}$ and $\vec{y}$ be spacelike vectors in $I R_{1}^{3}$ that span a spacelike vector subspace. Then there is a real number $\theta$ such that $<\vec{x}, \vec{y}>=\|\vec{x}\|\|\vec{y}\| \cos \theta$. This number is called the spacelike angle between the vectors $\vec{x}$ and $\vec{y}$. 
iv) Lorentzian timelike angle: Let $\vec{x}$ be a spacelike vector and $\vec{y}$ be a timelike vector in $I R_{1}^{3}$. Then there is a unique real number $\theta$ such that $|<\vec{x}, \vec{y}>|=\|\vec{x}\|\|\vec{y}\| \sinh \theta$. This number is called the Lorentzian timelike angle between the vectors $\vec{x}$ and $\vec{y}$.

Definition 2.2. ([1]) The Lorentzian casual character of a surface is defined by the aid of the induced metric on the surface. If this metric is a Lorentz metric, then the surface is said to be timelike. If the induced metric is a positive definite Riemannian metric, then the surface is said to be spacelike. This classification gives that the normal vector on the spacelike (timelike) surface is a timelike (spacelike) vector.

Lemma 2.1. ([5]) In the Minkowski 3space $I R_{1}^{3}$, the following properties are satisfied:

i) Two timelike vectors are never orthogonal.

ii) Two null vectors are orthogonal if and only if they are linearly dependent.

iii) A timelike vector is never orthogonal to a null (lightlike) vector.

\section{Spacelike Ruled Surfaces in the Minkowski 3-space}

Let $I$ be an open interval in the real line $I R, \vec{k}=\vec{k}(u)$ be a spacelike curve in $I R_{1}^{3}$ defined on $I$ and $\vec{q}=\vec{q}(u)$ be a unit direction vector of an oriented spacelike line in $I R_{1}^{3}$. Assume that $\langle\dot{\vec{q}}, \dot{\vec{q}}\rangle<0$ where $\dot{\vec{q}}=\frac{d \vec{q}}{d u}$. Then we have the following parametrization for a spacelike ruled surface $M$,

$$
\vec{r}(u, v)=\vec{k}(u)+v \vec{q}(u),
$$

where $\quad\langle\dot{\vec{k}}, \dot{\vec{k}}\rangle>0,\langle\vec{q}, \vec{q}\rangle=1 \quad$ and

$\dot{\vec{k}}=\frac{d \vec{k}}{d u}$

A parametric $u$-curve of this surface is a straight spacelike line of the surface which is called ruling. For $v=0$, the parametric $v$-curve of this surface is $\vec{k}=\vec{k}(u)$ which is called base curve or generating curve of the surface. In particular, if the direction of $\vec{q}$ is constant, the ruled surface is said to be cylindrical, and non-cylindrical otherwise.

The distribution parameter (or drall) of the spacelike ruled surface (1) is given by

$$
d=\frac{|\dot{\vec{k}}, \vec{q}, \dot{\vec{q}}|}{\langle\dot{\vec{q}}, \dot{\vec{q}}\rangle},
$$

If $|\dot{\vec{k}}, \vec{q}, \dot{\vec{q}}|=0$, then the normal vectors of the spacelike ruled surface are collinear at all points of the same ruling and at nonsingular points of the ruled surface $M$, tangent planes are identical. We then say that the tangent plane contacts the surface along a ruling. Such a ruling is called a torsal ruling. If $|\overrightarrow{\vec{k}}, \vec{q}, \dot{\vec{q}}| \neq 0$, then the tangent planes of the ruled surface $M$ are distinct at all points of the same ruling which is called nontorsal (Fig. 1, [12]). 


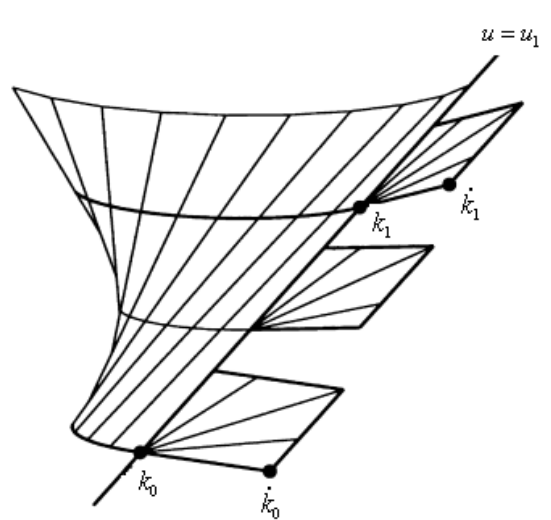

Figure 1. Distinct tangent planes along a non-torsal ruling $u=u_{1}$

Definition 3.1. A spacelike ruled surface whose all rulings are torsal is called a developable spacelike ruled surface. The remaining spacelike ruled surfaces are called skew spacelike ruled surfaces. Then from (2) it is seen that a spacelike ruled surface is developable if and only if at all its points the distribution parameter $d=0$.

For the unit normal vector $\vec{m}$ of the spacelike ruled surface, we have

$$
\begin{aligned}
\vec{m} & =\frac{\vec{r}_{u} \times \vec{r}_{v}}{\left\|\vec{r}_{u} \times \vec{r}_{v}\right\|} \\
& =\frac{(\dot{\vec{k}}+v \dot{\vec{q}}) \times \vec{q}}{\sqrt{\langle\dot{\vec{k}}, \vec{q}\rangle^{2}-\langle\dot{\vec{k}}+v \dot{\vec{q}}, \dot{\vec{k}}+v \dot{\vec{q}}\rangle \mid}}
\end{aligned}
$$

where $\dot{\vec{k}}=\frac{d \vec{k}}{d u}, \dot{\vec{q}}=\frac{d \vec{q}}{d u}$. From (3), at the points of a nontorsal ruling $u=u_{1}$ we have

$$
\vec{a}=\lim _{v \rightarrow \pm \infty} m\left(u_{1}, v\right)=\frac{\dot{\vec{q}} \times \vec{q}}{\|\dot{\vec{q}}\|}
$$

The plane $\alpha$ passing through the ruling $u_{1}$ and is perpendicular to the vector $\vec{a}$ is called the asymptotic plane which is the tangent plane at infinity. The tangent plane $\gamma$ passing through the ruling $u_{1}$ and perpendicular to the asymptotic plane $\alpha$ is called the central plane. The point $C$ where $\gamma$ is perpendicular to $\alpha$ is called central point of the ruling $u_{1}$ (Fig. 2). The set of central points of all the rulings of a spacelike ruled surface is called the striction curve of the surface. The straight lines which pass through point $C$ and are perpendicular to the planes $\alpha$ and $\gamma$ are called central tangent and central normal, respectively. Here, the tangent plane $\gamma$ is a spacelike plane and the asymptotic plane $\alpha$ is a timelike plane.

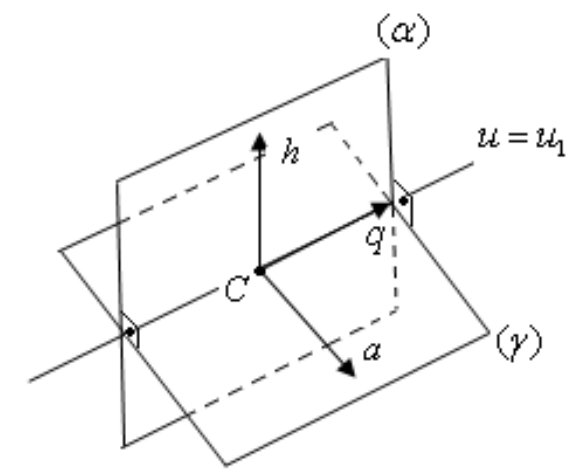

Figure 2. Asymptotic plane and central plane

Since the vectors $\vec{q}$ and $\dot{\vec{q}}$ are perpendicular, using (4) the representation of unit timelike central normal vector $\vec{h}$ is given by

$$
\vec{h}=\frac{\dot{\vec{q}}}{\|\dot{\vec{q}}\|}
$$


Substituting the parameter $v$ of the central point $C$ into equation (3), we get $\vec{h} \times \vec{m}=0$ which gives

$$
\begin{aligned}
\dot{\vec{q}} \times[(\dot{\vec{k}}+v \dot{\vec{q}}) \times \vec{q}] & =\langle\dot{\vec{q}}, \dot{\vec{k}}\rangle+v\langle\dot{\vec{q}}, \dot{\vec{q}}\rangle \\
& =\overrightarrow{0}
\end{aligned}
$$

From (6) we obtain

$$
v=-\frac{\langle\dot{\vec{q}}, \dot{\vec{k}}\rangle}{\langle\dot{\vec{q}}, \dot{\vec{q}}\rangle} .
$$

Thus, the parametrization of the striction curve $\vec{c}=\vec{c}(u)$ on a spacelike ruled surface is given by

$$
\vec{c}(u)=\vec{k}(u)+v \vec{q}(u)=\vec{k}-\frac{\langle\dot{\vec{q}}, \dot{\vec{k}}\rangle}{\langle\dot{\vec{q}}, \dot{\vec{q}}\rangle} \vec{q}
$$

So that, the base curve of the spacelike ruled surface is its striction curve if and only if $\langle\dot{\vec{q}}, \dot{\vec{k}}\rangle=0$.

Let us pay attention to the geometrical interpretation of the distribution parameter. Let the generating curve of a spacelike ruled surface be its striction curve and let the unnormed normal vector of the surface at striction point $(u, 0)$ be $\vec{m}_{0}$. By equality (3), we get

$$
\vec{m}_{0}=\dot{\vec{k}} \times \vec{q}
$$

Since $\vec{h} \times \vec{m}_{0}=0$, we obtain

$$
\dot{\vec{k}} \times \vec{q}=\beta \dot{\vec{q}}
$$

where $\beta=\beta(u)$ is a scalar function. This implies that

$$
\langle\dot{\vec{k}} \times \vec{q}, \dot{\vec{q}}\rangle=\beta\langle\dot{\vec{q}}, \dot{\vec{q}}\rangle
$$

Hence (2) yields $\beta=d$ and finally, $\dot{\vec{k}} \times \vec{q}=d \dot{\vec{q}}$. For $v \rightarrow \infty$, the normal vector is $\vec{m}_{\infty}=\dot{\vec{q}} \times \vec{q}$ and from (10) it is clear that $\vec{m}_{0} \perp \vec{m}_{\infty}$. By (3) the unnormed normal vector of the ruled surface is

$$
\overrightarrow{\vec{m}}=(\dot{\vec{k}} \times \vec{q})+v(\dot{\vec{q}} \times \vec{q})=\vec{m}_{0}+v \vec{m}_{\infty}
$$

If $\theta$ is the hyperbolic angle between $\vec{m}$ and $\vec{m}_{0}$ we have

$$
\left\{\begin{array}{l}
\left\langle\overrightarrow{\bar{m}}, \vec{m}_{0}\right\rangle=-\|\overrightarrow{\bar{m}}\|\left\|\vec{m}_{0}\right\| \cosh \theta, \\
\left\langle\overrightarrow{\bar{m}}, \vec{m}_{\infty}\right\rangle=\|\overrightarrow{\bar{m}}\|\left\|\vec{m}_{\infty}\right\| \sinh \theta .
\end{array}\right.
$$

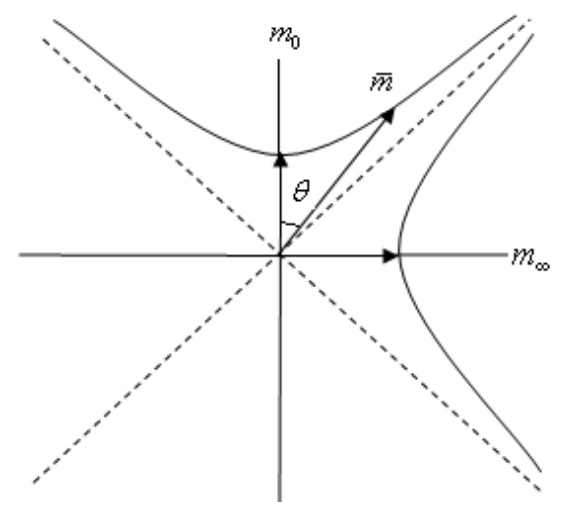

Figure 3. Hyperbolic angle $\theta$ between $\bar{m}$ and $m_{0}$

From (13) we get

$$
\tanh \theta=\frac{v}{d}
$$


So that, we give the following theorem which is known as Chasles theorem for spacelike ruled surfaces.

Theorem 3.1. Let the base curve of the spacelike ruled surface be its striction curve. For the angle $\theta$ between the tangent plane of the surface at the point $(u, v)$ of a nontorsal ruling $u$ and the central plane, we have

$$
\tanh \theta=\frac{v}{d}
$$

where $d$ is the distribution parameter of the ruling $u$ and the central point has the coordinates $(u, 0)$.

If $v=0$ i.e., the base curve of a spacelike ruled surface is also striction curve, then from (14) we have $\theta=0$. It means that tangent plane and central plane of the spacelike ruled surface are overlap.

\section{Frenet Equations and Frenet} Invariants of Spacelike Ruled Surfaces Let $\{C ; \vec{q}, \vec{h}, \vec{a}\}$ be an orthonormal frame of the spacelike ruled surface (Fig. 4). Here $C$ is the central point and $\vec{q}, \vec{h}, \vec{a}$ are the unit vectors of the ruling, the central normal and the central tangent, respectively. This frame is called the Frenet frame of the spacelike ruled surface $M$, and for this frame we have

$$
\begin{aligned}
& \vec{q} \times \vec{h}=-\vec{a}, \quad \vec{h} \times \vec{a}=-\vec{q}, \\
& \vec{a} \times \vec{q}=\vec{h}
\end{aligned}
$$

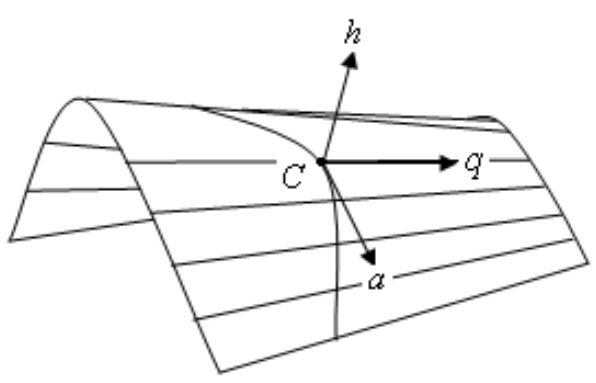

Figure 4. Frenet vectors of spacelike ruled surface

The set of all bound spacelike vectors $\vec{q}(u)$ at the point origin $O$ constitutes a cone which is called directing spacelike cone of the spacelike ruled surface $M$. The end points of the spacelike vectors $\vec{q}(u)$ drive a spherical timelike curve $k_{1}$ on Lorentzian unit sphere $S_{1}^{2}$ and this curve is called the Lorentzian spherical image of $M$, whose arc length is denoted by $S_{1}$.

Let now define the Frenet frame of the directing cone as the orthonormal frame $\{O ; \vec{q}, \vec{n}, \vec{z}\}$ where

$$
\vec{n}=\frac{d \vec{q}}{d s_{1}}=\vec{q}^{\prime}
$$

Since we have

$$
\vec{q}^{\prime}=\frac{\dot{\vec{q}}}{\|\dot{\vec{q}}\|}=\vec{h},
$$

by the aid of equation (15), we see that the tangent planes of the directing cone are parallel to the asymptotic planes of the spacelike ruled surface. Finally, we have

$$
\vec{z}=-\vec{q} \times \vec{h}=\vec{a}
$$


From (16), (17) and (18), we have the following theorem:

Theorem 4.1. The directing spacelike cone has the same Frenet frame with spacelike ruled surface $M$.

Let now compute the derivatives of the vectors $\vec{h}$ and $\vec{a}$ with respect to the arc length parameter $s_{1}$. Since $\vec{h}$ is central normal vector of spacelike ruled surface $M$, by Definition 2.2, we have $\langle\vec{h}, \vec{h}\rangle=-1, \quad$ thus $\quad\left\langle\vec{h}, \vec{h}^{\prime}\right\rangle=0$. Consequently,

$$
\vec{h}^{\prime}=b_{1} \vec{q}+b_{2} \vec{a}
$$

From $\langle\vec{h}, \vec{q}\rangle=0$, it follows that

$$
\left\langle\vec{h}^{\prime}, \vec{q}\right\rangle+\left\langle\vec{h}, \vec{q}^{\prime}\right\rangle=b_{1}-1=0,
$$

and if we put $b_{2}=\kappa$ we get

$$
\vec{h}^{\prime}=\vec{q}+\kappa \vec{a},
$$

where $\kappa$ is called the conical curvature of the directing cone. From $\langle\vec{h}, \vec{a}\rangle=0$, we have

$$
\left\langle\vec{h}^{\prime}, \vec{a}\right\rangle+\left\langle\vec{h}, \vec{a}^{\prime}\right\rangle=\kappa+\left\langle\vec{h}, \vec{a}^{\prime}\right\rangle=0
$$

which gives

$$
\left\langle\vec{h}, \vec{a}^{\prime}\right\rangle=-\kappa
$$

Further, from the equalities $\langle\vec{a}, \vec{a}\rangle=1$ and $\langle\vec{a}, \vec{q}\rangle=0$ it follows $\left\langle\vec{a}, \vec{a}^{\prime}\right\rangle=0$ and

$$
\left\langle\vec{a}^{\prime}, \vec{q}\right\rangle+\left\langle\vec{a}, \vec{q}^{\prime}\right\rangle=\left\langle\vec{a}^{\prime}, \vec{q}\right\rangle=0,
$$

respectively, which means that the vector $\vec{a}^{\prime}$ is collinear with the timelike vector $\vec{h}$, i.e. $\vec{a}^{\prime}=b_{3} \vec{h}$ where $b_{3}=b_{3}\left(s_{1}\right)$. By the equality (23) we get

$$
\left\langle\vec{h}, \vec{a}^{\prime}\right\rangle=-b_{3}=-\kappa,
$$

and thus

$$
\vec{a}^{\prime}=\kappa \vec{h}
$$

For the Lorentzian spherical curve $k_{3}$ with arc length $s_{3}$ circumscribed on Lorentzian unit sphere $S_{1}^{2}$ by the bound vector $\vec{a}$ at the point $O$, we have

$$
\frac{d s_{3}}{d s_{1}}=\left\|\vec{a}^{\prime}\right\|=\kappa .
$$

where $\kappa$ is the conical curvature of the directing cone. Thus, with (17), (21) and (26) we have the following theorem.

Theorem 4.2. The Frenet formulas of spacelike ruled surface $M$ and of its directing spacelike cone with respect to the arc length parameter $s_{1}$ are given by

$$
\left[\begin{array}{l}
d \vec{q} / d s_{1} \\
d \vec{h} / d s_{1} \\
d \vec{a} / d s_{1}
\end{array}\right]=\left[\begin{array}{lll}
0 & 1 & 0 \\
1 & 0 & \kappa \\
0 & \kappa & 0
\end{array}\right]\left[\begin{array}{l}
\vec{q} \\
\vec{h} \\
\vec{a}
\end{array}\right] .
$$

From (28) the Darboux vector (instantaneous rotation vector) of the Frenet frame $\{O ; \vec{q}, \vec{h}, \vec{a}\}$ can be given by $\vec{w}_{1}=-\kappa \vec{q}+\vec{a}$. Thus, for the derivatives in (28) we can write 
$\vec{q}^{\prime}=\vec{w}_{1} \times \vec{q}, \quad \vec{h}^{\prime}=\vec{w}_{1} \times \vec{h}, \quad \vec{a}^{\prime}=\vec{w}_{1} \times \vec{a}$.

Let $s$ be the arc length parameter of the striction curve $\vec{c}(s)$. Furthermore, we call $\frac{d s_{1}}{d s}=\kappa_{1}$ as the first curvature and $\frac{d s_{3}}{d s}=\kappa_{2}$ as the second curvature of the spacelike ruled surface $M$ or rather of its directing cone. Then we have

$$
\kappa_{2}=\kappa \kappa_{1} .
$$

Spacelike ruled surfaces for which $\kappa_{1} \kappa_{2} \neq 0$ and $\kappa=\left(\kappa_{2} / \kappa_{1}\right)=$ constant have a spacelike cone of revolution as their directing cones. If $\kappa_{1} \neq 0, \kappa_{2}=0$, then we obtain a directing spacelike plane instead of a spacelike directing cone and these spacelike ruled surfaces, satisfying $\kappa_{1} \neq 0, \kappa_{2}=0$, are called spacelike conoids.

Multiplying (28) by the first curvature $\frac{d s_{1}}{d s}=\kappa_{1}$, we have the following theorem.

Theorem 4.3. The Frenet formulas of the spacelike ruled surface $M$ and of its directing cone with respect to the arc length parameter of the striction curve are given by

$$
\left[\begin{array}{l}
d \vec{q} / d s \\
d \vec{h} / d s \\
d \vec{a} / d s
\end{array}\right]=\left[\begin{array}{ccc}
0 & \kappa_{1} & 0 \\
\kappa_{1} & 0 & \kappa_{2} \\
0 & \kappa_{2} & 0
\end{array}\right]\left[\begin{array}{l}
\vec{q} \\
\vec{h} \\
\vec{a}
\end{array}\right],
$$

where $\kappa_{1}=\frac{d s_{1}}{d s}, \quad \kappa_{2}=\frac{d s_{3}}{d s}$ and $s_{1}, s_{3}$ are arc lengths of spherical curves $k_{1}, k_{3}$ circumscribed by the bound vectors $\vec{q}$ and $\vec{a}$, respectively.

For the derivatives of vectors of the Frenet frames $\{O ; \vec{q}, \vec{h}, \vec{a}\}$ with respect to the arc length of striction curve of the surface, the Darboux vector can be given by $\vec{w}_{2}=-\kappa_{2} \vec{q}+\kappa_{1} \vec{a}$. Thus, the derivatives in (30) we can written as follows

$$
\begin{aligned}
& \frac{d \vec{q}}{d s}=\vec{w}_{2} \times \vec{q}, \quad \frac{d \vec{h}}{d s}=\vec{w}_{2} \times \vec{h}, \\
& \frac{d \vec{a}}{d s}=\vec{w}_{2} \times \vec{a}
\end{aligned}
$$

Now, we will show that the tangent of striction curve of the spacelike ruled surface at the central point $C$ is perpendicular to the central normal vector $\vec{h}$. From (8), we have

$$
\frac{d \vec{c}}{d u}=\dot{\vec{k}}+v \dot{\vec{q}}+\dot{v} \vec{q}
$$

and further by using (5) and (7) we get

$$
\begin{aligned}
\left\langle\vec{h}, \frac{d \vec{c}}{d u}\right\rangle & =\langle\vec{h}, \dot{\vec{k}}\rangle+v\langle\dot{\vec{q}}, h\rangle \\
& =\frac{\langle\dot{\vec{k}}, \dot{\vec{q}}\rangle}{\|\dot{\vec{q}}\|}-\frac{\langle\dot{\vec{k}}, \dot{\vec{q}}\rangle\langle\dot{\vec{q}}, \dot{\vec{q}}\rangle}{\|\dot{\vec{q}}\|\langle\dot{\vec{q}}, \dot{\vec{q}}\rangle}=0
\end{aligned}
$$

Let the angle $\varphi$ be the spacelike angle between the unit tangent vector $t$ of striction curve and the ruling $q$. Then we can write

$$
\vec{t}=\frac{d \vec{c}}{d s}=(\cos \varphi) \vec{q}+(\sin \varphi) \vec{a} .
$$


Thus, while the equation of the spacelike ruled surface is

$$
\vec{r}(s, v)=\vec{c}(s)+v \vec{q}(s)
$$

the equation of striction curve is

$$
\vec{c}(s)=\int[(\cos \varphi) \vec{q}+(\sin \varphi) \vec{a}] d s
$$

For the parameter of distribution, using (2) and (30), we have

$$
d=\frac{\left|\vec{q}, \frac{d \vec{q}}{d s}, \frac{d \vec{c}}{d s}\right|}{\left\langle\frac{d \vec{q}}{d s}, \frac{d \vec{q}}{d s}\right\rangle}=-\frac{|\vec{q}, \vec{h}, \vec{t}|}{\kappa_{1}} .
$$

From (31) and (34), it follows that

$$
\begin{aligned}
\sin \varphi & =\langle\vec{a}, \vec{t}\rangle=\langle-\vec{q} \times \vec{h}, \vec{t}\rangle \\
& =-|\vec{q}, \vec{h}, \vec{t}|=\kappa_{1} d
\end{aligned}
$$

By considering (31) and (35), the Frenet formulas of $M$ are given as follows

$$
\left\{\begin{array}{l}
\frac{d \vec{c}}{d s_{1}}=\frac{\cos \varphi}{\kappa_{1}} \vec{q}+\frac{\sin \varphi}{\kappa_{1}} \vec{a}=f \vec{q}+d \vec{a} \\
\frac{d \vec{q}}{d s_{1}}=\vec{h} \\
\frac{d \vec{h}}{d s_{1}}=\vec{q}+\kappa \vec{a} \\
\frac{d \vec{a}}{d s_{1}}=\kappa \vec{h}
\end{array}\right.
$$

The functions $f\left(s_{1}\right), d\left(s_{1}\right), \kappa\left(s_{1}\right)$ are the invariants of the spacelike ruled surface $M$. They determine the spacelike ruled surface uniquely up to its position in the space.
5. Example (Conoid of the $1^{\text {st }}$ kind). Let consider the ruled surface $M$ defined by

$$
\begin{aligned}
& \vec{r}(u, v)=(v \sinh u, v \cosh u, u), \\
& (-1<v<1) .
\end{aligned}
$$

This parametrization defines a noncylindrical spacelike ruled surface which is said to be a conoid of the $1^{\text {st }}$ kind in $I R_{1}^{3}$ (Fig. 5) [8]. The base curve and the ruling of $M$ are $\vec{k}(u)=(0,0, u)$ and $\vec{q}(u)=(\sinh u, \cosh u, 0)$, respectively. The distribution parameter of $M$ is $d=1$. So that, the surface $M$ is a skew spacelike ruled surface. The striction curve of $M$ is given by

$$
\vec{c}(u)=\vec{k}(u)=(0,0, u) .
$$

The arc length parameter of the striction curve is $u=s$. Thus, the striction curve and Frenet vectors of $M$ with respect to the arc length parameter $s$ are

$$
\begin{aligned}
& \vec{c}(s)=(0,0, s), \\
& \vec{q}(s)=(\sinh s, \cosh s, 0), \\
& \vec{h}(s)=(-\cosh s,-\sinh s, 0), \\
& \vec{a}(s)=(0,0,1) .
\end{aligned}
$$

The derivative formulas with respect to the arc length parameter $s$ are

$$
\left[\begin{array}{l}
d \vec{q} / d s \\
d \vec{h} / d s \\
d \vec{a} / d s
\end{array}\right]=\left[\begin{array}{ccc}
0 & -1 & 0 \\
-1 & 0 & 0 \\
0 & 0 & 0
\end{array}\right]\left[\begin{array}{l}
\vec{q} \\
\vec{h} \\
\vec{a}
\end{array}\right]
$$

and the first and second curvatures of spacelike ruled surface are $\kappa_{1}=-1$ and $\kappa_{2}=0$, respectively. By (29), conical curvature is $\kappa=0$. From (35), for the angle $\varphi$ we have $\varphi=3 \pi / 2$. So that, $f=0$. Consequently, the Frenet 
invariants are obtained as $f=0, \kappa=0$ ,$d=1$.

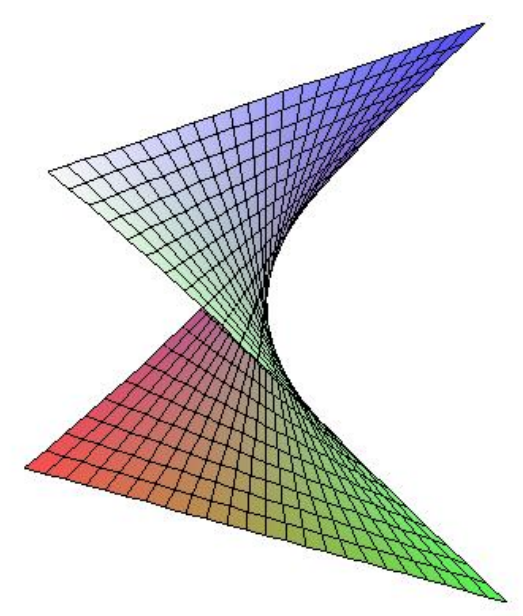

Figure 5. A conoid of the $1^{\text {st }}$ kind

\section{References}

[1] Beem, J.K., Ehrlich, P.E. 1981. Global Lorentzian Geometry. Marcel Dekker, New York.

[2] Dillen, F., Sodsiri, W. 2005. Ruled surfaces of Weingarten type in Minkowski 3-space: J. Geom., Vol. 83, No. (1-2), pp. 10-21. DOI:10.1007/s00022-005-0002-4

[3] Ekici, C., Özüsağlam, E. 2012. On the Method of Determination of a Developable Timelike Ruled Surface: KJSE- Kuwait Journal of Science \& Engineering, Vol. 39(1A), pp. 19-41.

[4] Ekici, C., Öztürk, H. 2013. On Timelike Ruled Surfaces in Minkowski 3Space: Universal Journal of Applied Science, Vol. 1, No. 2, pp. 56-63. DOI: 10.13189/ujas.2013.010205

[5] Greub, W. 1975. Linear Algebra, Fourth ed., Springer-Verlag, New York.

[6] Guggenheimer, H. 1963. Differential Geometry, McGraw- Hill Book Comp. Inc. London, Lib. Cong. Cat. Card No. 68-12118.

[7] Karger, A. Novak, J. 1978. Space Kinematics and Lie Groups. STNL Publishers of Technical Lit., Prague, Czechoslovakia.
[8] Kim, Y.H., Yoon, W.D. 2004 Classification of ruled surfaces in Minkowski 3-space: Journal of Geometry and Physics, Vol. 49(1), pp. 89-100. DOI: 10.1016/S03930440(03)00084-6.

[9] Küçük, A. 2004. On the developable timelike trajectory ruled surfaces in Lorentz 3-space $I R_{1}^{3}$ : App. Math. Comput., Vol. 157(2), pp. 483-489. DOI: $10.1016 /$ j.amc.2003.09.001.

[10] O'Neill, B. 1983. Semi-Riemannian Geometry with Applications to Relativity. Academic Press, London.

[11] Önder, M., Uğurlu, H.H. 2013. Frenet Frames and Invariants of Timelike Ruled Surfaces: Ain Shams Eng J., Vol. 4, pp. 507-513. DOI: 10.1016/j.asej.2012.10.003.

[12] Peternel, M., Pottmann, H., Ravani, B. 1999. On the computational geometry of ruled surfaces: Comp. Aided Geom. Design, Vol. 31, pp. 1732 DOI: $10.1016 / \mathrm{S} 0010-$ 4485(98)00077-3.

[13] Ratcliffe, J.G. 2006. Foundations of Hyperbolic Manifolds, Springer.

[14] Ravani, B., Ku, T.S. 1991. Bertrand Offsets of ruled and developable surfaces: Comp. Aided Geom. Design, Vol. 23, No. 2, pp. 147-152. DOI: 10.1016/0010-4485(91)90005-H

[15] Turgut, A,, Hacısalihoğlu, H.H. 1997. Timelike ruled surfaces in the Minkowski 3-space: Far East J. Math. Sci., Vol. 5, No. 1, pp. 83-90.

[16] Uğurlu, H.H., Çalışskan, A. 2012. Darboux Ani Dönme Vektörleri ile Spacelike ve Timelike Yüzeyler Geometrisi. Celal Bayar Üniversitesi Yayınları, Yayın No: 0006.

[17] Wang, D.L., Liu, J., Xiao, D.Z. 1997. Kinematic Differential Geometry of a Rigid Body in Spatial Motion I-A New Adjoin Approach and Instantaneous Properties of a Point Trajectory in Spatial Kinematics: Mech. and Mach. Theory, Vol. 32, No. 4, pp. 419-432. 
DOI:

114X(96)00075-4

[18] Wang, D.L., Liu, J., Xiao, D.Z. 1997. Kinematic Differential Geometry of a Rigid Body in Spatial Motion II-A New Adjoin Approach and Instantaneous Properties of a Line Trajectory in Spatial Kinematics: Mech. and Mach. Theory, Vol. 32, No. 4, pp. 433-444. DOI: 10.1016/S0094114X(96)00076-6.
[19] Wang, D.L., Liu, J., Xiao, D.Z. 1997. Kinematic Differential Geometry of a Rigid Body in Spatial Motion IIIDistribution of Characteristic Lines in the Moving Body in Spatial Motion: Mech. and Mach. Theory, Vol. 32, No. 4, pp. 445-457. DOI: 10.1016/S0094-114X(96)00077-8. 